\title{
PRINCIPIA IURIS DE LUIGI FERRAJOLI *
}

\author{
Mauro Palma \\ Presidente del Comité Europeo para la Prevención de la Tortura \\ (Consejo de Europa)
}

RESUMEN. El texto desarrolla dos observaciones y evidencia, sucesivamente, algunos puntos críticos.

La primera observación es que la construcción propuesta tiene fundamento en la afirmación que señala una intrínseca interdependencia entre la democracia y el Derecho y entre el Derecho y la razón: de aquí nace la elección de una teoría axiomatizada del Derecho. El sistema axiomático formal, por tanto, no es parte accesoria, eliminable de la obra; es, por el contrario, su intrínseca estructura y razón.

La segunda observación es que la estructura que surge es la de un «constitucionalismo fuerte" que identifica un sistema de límites y también de obligaciones al poder, que sin ellas sería soberano, de la mayoría.

A partir de estas observaciones preliminares, se analizan algunos puntos. El primero reside en el carácter intrínsecamente inacabado del sistema, característica que FERRAJOLI presenta como un valor adjunto. El segundo es la relación de la formalización con la explicabilidad del lenguaje y, por tanto, con la comunicabilidad del Derecho. El tercero es el doble rol del sistema axiomático: definir un posible universo de discurso o describir rigurosamente un universo ya definido de otro modo. El último nodo es la aplicabilidad del método propuesto a los sistemas de Derecho contemporáneo, fuertemente influenciados por el contacto directo con los sistemas del common law.

Palabras clave: Ferrajol, Principia iuris, teoría axiomatizada del Derecho, constitucionalismo

ABSTRACT. The text develops two observations and then highlights some critical points.

First observation: the proposed construction has a cornerstone in the affirmation of the intrinsic interdependence between democracy and law and between law and reason; hereof comes the choice of an axiomatic theory of law. Therefore the formal axiomatic system is not an additional and eliminable part of the work but its intrinsic structure and reason.

Second: the structure that emerges is of a "strong constitutionalism", which outlines a system of obligations and limits to the power of the majority, otherwise unlimited.

Starting from these preliminary observations, some critical points are discussed. The first point lies in the inherently incompleteness of the system, although such a characteristic is seen by FERRAJOLI as an additional value of the system. The second is the relationship between formalization and understandability of language, and then between formalization and communicability of law. The third point is the dual role of an axiomatic system: to define a possible universe of discourse, or to describe a universe already strictly defined otherwise. Finally -that is the last critical point discussed in the text- the applicability of the proposed method to contemporary legal systems, strongly influenced by direct contact with the systems of common law.

Keywords: FerRajol, Principia luris, axiomatic theory of law, constitutionalism.

\footnotetext{
* Fecha de recepción: 1 de diiembre de 2008. Fecha de aceptación: 18 de diciembre de 2008.
} 


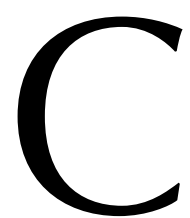

onfieso que he tenido algunas dificultades en dar una dirección a estas breves observaciones.

La primera dificultad radica en tener que enfrentar la sistematicidad y la globalidad de una obra que no permite aproximaciones parciales, sin contextualizarlas, como se debería, en aquel espacio de discurso que ésta define con absoluta precisión. Un obra que reconstruye el espacio de las normas, de las acciones, de los efectos, de las garantías, de los derechos individuales y sociales y de las obligaciones que ellos implican, que - como se afirma explícitamente- ve en el Derecho el fulcro de la construcción de la democracia y en la razón la esencia constitutiva del Derecho. Una obra global que se mueve en el trazado iluminista, en el primado absoluto de la razón y que en este sentido, si bien pesimista en el análisis del presente y en someter a criterios de racionalidad y de consistencia lógica las nuevas prácticas y los nuevos temas del Derecho concreto, es sustancialmente optimista porque tiene fe en la posibilidad de un criterio regulador y reorganizador de la complejidad.

La sistematicidad de esta obra es un desafío también para quien ha tenido la posibilidad, en el curso de los años que han acompañado su construcción, de hablar, de vez en cuando, con el autor además de gozar del raro privilegio de no sentir miedo o repulsión delante del uso recurrente de formalizaciones y símbolos.

La segunda dificultad tiene una raíz estrictamente biográfica: he abandonado la lógica, en particular la deóntica de la cual proviene mi formación. Y, al mismo tiempo, de formación no jurídica, he acentuado la frecuentación jurídica, hasta asumir la coordinación de los trabajos de un Comité compuesto sobre todo por juristas: el Comité europeo encargado de controlar el respeto de las obligaciones que tienen los Estados que derivan del art. 3 de la Convención europea por la salvaguarda de los derechos del hombre y de las libertades fundamentales. Esta segunda dificultad me hace sentir extranjero en las dos áreas: la lógica y el Derecho, no obstante a la relación existencial que tengo con ambas.

Una tercera dificultad nace de la connotación específica de mi trabajo actual, de constante monitoraje a lugares opacos como son los de la privación de la libertad, que me lleva a confrontarme con la materialidad espuria del ejercicio y del no ejercicio del Derecho: un trabajo «en el Derecho» que por lo tal difícilmente se relaciona con el rigor lógico y definitorio de la obra de FERRAJOLI la cual sustancialmente constituye un trabajo «sobre el Derecho». El trabajo «en el Derecho» está fuertemente radicado en la materialidad de la aplicación, está marcado en su corpus teórico por la efectividad de los éxitos de lo afirmado y establecido; el trabajo «sobre el Derecho» está depurado de tal materialidad e indica los principios y las líneas asintóticas hacia las cuales aquellas actualizaciones deberían convergir.

En el Derecho penal empírico, en el cual me muevo, aquel de los lugares poco transparentes en donde la práctica de justicia tiene su punto de llegada y la sanción se manifiesta en concreción y cotidianidad, en materialidad que se hace sufrimiento, frecuentemente ignominia, tortura, la falacia no es lógica, es factual, y como tal es inasible.

Con este múltiple sentimiento - la fascinación por la construcción lógica, el compartir de la centralidad de la razón y del Derecho en la construcción de la democracia 
moderna y el eco de mi experiencia de verífica del Derecho concreto- me aventuré en la lectura de la obra de FERRAJOLI, desarrollando preliminarmente algunas observaciones y sucesivamente evidenciando algunos puntos críticos que quisiera compartir con los autores y los demás lectores fascinados.

La primera observación, a la que ya he hecho referencia, es que la construcción propuesta tiene su fundamento en la afirmación que señala una intrínseca interdependencia entre la democracia y el Derecho y entre el Derecho y la razón. De aquí nace la elección metodológica de proponer una teoría axiomatizada del Derecho, así come se configura en los países que en la segunda postguerra se dieron una Constitución escrita, rígida y garantizada. La teoría axiomatizada es funcional a una teoría, de la democracia constitucional, no axiomatizada, pero escandida sobre la base de la axiomatización de la anterior. La democracia es la expresión empírica del implanto formal, es su equivalente isomorfa y no simplemente un conjunto de prácticas de buen gobierno. La democracia es tal, si no traiciona este isomorfismo.

A esta elección de método FERRAJOLI atribuye una «una función práctica», no simplemente la de dar una reconstrucción pulida, depurada de falacias y, por ello coherente, como si se tratara de una organización lógica de material que en lo concreto ha sido definida y que requiere una sistematización ex post. El de hecho escribe que «el método axiomático representa [...] un poderoso instrumento de clarificación conceptual, de elaboración sistemática y racional, de análisis crítico y —se preste atención— de invención teórica, entonces, particularmente eficaz para la explicación de la creciente complejidad e inefectividad de los ordenamientos modernos» ${ }^{1}$.

El sistema axiomático formal —y esta es la segunda observación — no es parte accesoria, eliminable de la obra, es su intrínseca estructura y razón. De hecho, si es verdad que los pasos del cálculo son facultativos, así sean de simple lectura y útiles para volver transparentes las afirmaciones del texto, es también verdad que la necesidad y la existencia de cálculo son connotaciones estructurantes de la obra. El autor lo comprueba a través de dos distinciones deónticas entre «validez» y «vigor» de las leyes y entre «vigor»y «efectividad» del Derecho en las prácticas institucionales. La reducción de tales distinciones es el principio que da forma a la obra. A partir de esto él desarrolla tanto la crítica al Derecho ilegítimo, es decir en contraste con la Constitución, como la elaboración de las garantías en cuanto técnicas para reducir la inefectividad de las promesas constitucionales.

La tercera observación que quiero resaltar se refiere al impacto de la obra, oportunamente definido «ius-constitucionalista» o también de «fuerte constitucionalismo» —y no por casualidad no se evita el uso de la expresión «neo-constitucionalismo» para evitar el aspecto de superposición de planos éticos y planos normativos que esta expresión trae consigo- - Las Constituciones de la segunda postguerra, en general, y de la italiana en particular — observa el autor - modifican la estructura del Derecho porque impiden la producción de normas invalidas en cuanto en contradicción con los derechos de libertad e imponen la producción de leyes de actuación idóneas para garantizar los derechos sociales. Estas leyes constituyen entonces un sistema de límites y también de obligaciones al poder, que sin ellas sería soberano de la mayoría. Aquí reside la

\footnotetext{
${ }^{1}$ S. MARIETTI, enero 2008: «Intervista a Luigi Ferrajoli», en Reset, n. ${ }^{\circ} 105$.
} 
relación con la democracia, relación que es problemática sólo si se interpreta a la democracia como omnipotencia de la mayoría, es decir «democracia política»; mientras no lo es si es entendida como garantía de derechos fundamentales para todos, es decir como «democracia constitucional».

Una última observación preliminar se relaciona con el procedimiento del cálculo: el cálculo funciona, se desarrolla coherentemente y con precisión. Algunos elementos de «ingenuidad» lógica que pueden ser resaltados tienen que ver con la mayor o menor opacidad del implanto, que en parte no corresponde a criterios de lógica deóntica - más bien a criterios de lógica predicativa de orden I con la añadidura de operadores cerca de la posibilidad y la necesidad - pero el implanto funciona, no presenta falacias y su simplicidad es su elemento de fuerza.

A partir de estas observaciones preliminares, se hacen evidentes los puntos a los que me he referido anteriormente.

El primer punto, que considero no resuelto aún, reside en el carácter intrínsecamente inacabado del sistema. ¿Puede, de hecho, un sistema como el propuesto ser completo? En términos generales, creo que la respuesta sea negativa, porque considero que de no ser así sería el fin de la historia.

Sabemos que los sistemas lógicos no pueden huir a dos criterios: deben de ser coherentes y completos. Son dos principios impuestos al legislador.

Es simple aceptar el carácter coherente para los sistemas que describen actos de expresión y/o comportamiento, como los sistemas lingüísticos o el sistema penal. De hecho, en este último, un comportamiento no debe ser calificable al mismo tiempo como lícito o como no lícito, ni un acto derivado de un comportamiento lícito no puede ser calificado como ilícito.

La característica que se refiere a lo completo del sistema es menos lineal porque se manifiesta bajo dos diversos aspectos. El primer aspecto se refiere a que una conducta no puede ser deónticamente incalificada, el segundo aspecto se refiere a que si se dan relaciones lógicas entre normas, el legislador debe desarrollar todas las implicaciones lógicas de orden superior. Como consecuencia se establecen dos posibles hipótesis de lagunas en los sistemas normativos, la ausente connotación deóntica de una conducta y la ausencia de la emanación de una norma que haga efectiva otra norma.

FERRAJOLI no niega el riesgo del carácter incompleto, al contrario lo presenta como un valor adjunto, cuando en las primeras páginas da cuenta de la elección de un método axiomático: «Se entiende que una teoría construida de esta manera es como un mosaico, o si se prefiere un complicado edificio del que primero se deben construir los fundamentos, después levantar los muros maestros y sólo sucesivamente construir las paredes divisorias y preparar las decoraciones internas. Con la diferencia que en el trabajo teórico no se dispone desde el comienzo ni del plano ni de los materiales de construcción [...] un diseño inicial existe [...] una teoría del Estado constitucional de Derecho y de las técnicas de garantía de derechos subjetivos, comenzando por aquellos fundamentales» ${ }^{2}$. De aquí la apertura a descubrimientos sucesivos que él da a su trabajo.

\footnotetext{
${ }^{2}$ L. Ferrajoli, 2007: Principia Iuris, Bari: G. Laterza editore, vol. I, pp. 55, 56.
} 
Aquí nace una hipótesis que yo veo detrás de los Principia Iuris: el sistema propuesto parece motivado también y no incidentalmente, por una exigencia político- normativa, por un gran deseo de someter a verificación la producción de las normas; de poner orden, freno y verificabilidad lógica, a interpretaciones erróneas. En pocas palabras de diferenciar aquello que es lógicamente deducible de aquello que no lo es, se trata de un gran filtro que somete a verificación de coherencia muchos temas, elementos impuros entrados en las normas: aporías, elecciones hechas sobre la base de criterios de oportunidad y de gastabilidad política y no de fundamentabilidad jurídica y de coherencia democrática. Y no es secundario que los temas expuestos en el segundo volumen, los más susceptibles de conmixtión se expongan en el interior del esquema construido de definiciones de democracia constitucional, depurándolo de falsedades semánticas o de pliegues adquiridos en el debate político: esto se hace evidente sobre todo en la parte dedicada a los derechos sociales.

En resistematizar y depurar cuanto es producido, a través del examen lógico definido, FERRAJOLI es EUCLIDES, y no tanto HILBERT: el trabajo del primero se dedica a dar forma y a sistematizar lógicamente el material precedente, lo que se ha producido que se conoce pero que no ha sido sistematizado; el trabajo del segundo se dedica a dar expresión formal depurada de referencias experienciales para así abrir el camino a otras interpretaciones posibles del mismo esquema formal construido.

FERRAJOLI sigue más al griego y que al alemán. Así se hable del desafío de inventar —el sistema «solicita la imaginación y la invención teórica»— es evidente que su interés es someter a un análisis riguroso las eventuales invenciones más que formular nuevas. No se trata de construir un nuevo derecho, como para el geómetra no se trata de construir un nuevo espacio, se trata mas bien de entender si nuevos elementos son coherentes con la totalidad del implanto y de ser así como.

Tampoco hay una libertad de «movimiento» lógico, de juego coherente, de verdad como combinación posible, de apertura hacia lo impredecible — que es una connotación lógica del novecientos-. Aquí en cambio el aparato al que se debe llegar es conocido en sus características constituyentes, el sistema está dado y se quiere demostrar su coherencia intrínseca y la incoherencia de otras elecciones - que se declaran parte de la misma opción democrática.

El segundo punto es la relación con la formalización. Se podría dar una estructura lógica sin formalizarla. ¿La acentuación lógica ayuda o desorienta? No es un problema de lectura: si el Derecho es el elemento estructurante del Estado constitucional y por ende de la esfera pública. ¿Cuál comunicación del Derecho se debe practicar?

A quien ha seguido desde lejos y durante años a FERRAJOLI, la elección de la formalización no sorprende: el Derecho es un sistema lógico formalizable y «tarea de la (metateoría) semántica de la teoría del Derecho es la identificación del conjunto de cosas y/o experiencias que forman el objeto de la teoría del Derecho y respecto al cual las tesis en ella formuladas pueden ser interpretadas y verificadas». Esta idea la anunciaba en su ensayo de $1983^{3}$.

${ }^{3}$ El ensayo se encuentra en el volumen colectivo de Estudios dedicado a N. BOBBio (compilado por U. SCARPELLI), 1983: La teoria generale del diritto. Problemi e tendenze attuali, Edizione Comunitá. 
La formalización escogida sigue, como ya dicho, las reglas de la lógica y sus simbolismos.

La discusión sobre la potencialidad informativa de la formalización es antigua. Bastará citar algunas de las polémicas que han acompañado el programa logicista relacionado a otra disciplina, que desde hace tiempo hace uso de instrumentos formales, la matemática.

De vez en cuando al lector desatento le pueden venir en mente, consultando el tercer volumen, algunas exclamaciones de H. POINCARÉ: «pero — escribía- si se requieren veintisiete ecuaciones para establecer que 1 es un número, ¿cuántas se necesitan para demostrar un verdadero teorema?» ${ }^{4}$. «La carcajada de POINCARÉ —escribe G. LOLLI, cuando cita la frase - resuena todavía hoy, devastadora, en contra de las pretensiones de escribir matemática en un lenguaje simbólico y se transmite en la invencible indolencia del matemático hacia todo aquello que tenga el sabor de lenguajes formales y de lógica». Él atacaba mientras los adversarios exultaban por la realización del sueño leibniziano de construir una lingua characterística y un asociado calculus ratiocinator —el famoso «icalculemos!»— atribuyendo a la formalización las ventajas a aquello que POINCARÉ veía como defecto: claridad, brevedad, precisión y mayor afidabilidad.

En este dilema nos encontramos aún hoy en día. ¿Qué es la claridad y la explicabilidad del lenguaje? ¿Es verdad que ella está garantizada por la autoevidencia del lenguaje formal, como en fondo parece que creyera FERRAJOLI?

Ciertamente en esta hipótesis ayuda R. CARNAP: «En el lenguaje simbólico a diferencia que en el ordinario lenguaje de palabras, tenemos signos que no son ambiguos y formulaciones que son exactas: por esto mismo en este lenguaje la pureza y lo correcto de una derivación puede controlarse con mayor facilidad y cuidado [...] Una derivación en un lenguaje de palabras comprende presupuestos que no se habían hecho explícitos, pero que habían entrado inadvertidamente. Una ulterior ventaja de usar símbolos artificiales en vez de palabras se revela en la concisión y en la claridad de las fórmulas simbólicas. Frecuentemente un enunciado que necesita muchas líneas en un lenguaje de palabras (por lo que su claridad es reducida) puede ser representado simbólicamente en una línea o menos» ${ }^{5}$.

Si le reconocemos al Derecho el rol de factor constitutivo, de constructor de la democracia y si reconocemos que el espacio público de la comunicación democrática se constituye como espacio de producción de reglas y normas, que no se relegan a la instancia privada del legislador, entonces el problema de la comunicación del Derecho viaja simultáneamente al problema de su coherencia lógica. Y mucho debe hacerse para que la coherencia no sea solamente un puro control lógico, pero un tejido que entrelaza el implanto lógico y el implanto factual.

El tercer punto es el del doble rol del sistema axiomático: definir un posible universo de discurso o describir rigurosamente un universo ya definido de otro modo. El pun-

\footnotetext{
${ }^{4}$ Citada por G. LoLLI, 1987: La Macchina e le dimostrazioni: Matematica, Logica, Informatica, Bologna: Il Mulino, y extraída de 1908: Les dernières efforts des logistiens.

${ }^{5}$ R. CARNAP, 1958: Introduction to Symbolic Logic and Its Applications, New York: Dover, p. 2.
} 
to es en síntesis, entre el sistema axiomático que define el mundo posible y un sistema axiomático que da orden a un mundo descrito de varias formas. Me he ya referido al hecho que FERRAJOLI niega fuertemente la posibilidad de ver el control lógico como un gesto expost al cual someter la producción normativa y el implanto del Derecho. Lo ve como un ex ante que debe dar forma a la producción misma; es un método productivo y no un criterio de análisis. Para FERRAJOLI la teoría procede mediante pruebas, errores y cambios gracias al método axiomático que permite los cambios de acción en el interior de un sistema lógicamente vinculado. «Reiteradamente —él afirma- he cambiado o integrado definiciones y postulados: naturalmente cada cambio de una tesis primitiva (postulado o definición) comporta la obligación de modificar la entera cadena de las tesis que de ella directa o indirectamente derivan». En este proceso reside la potencialidad explicativa del sistema y no su limitación.

Sin embargo la cuestión no se limita a una declaración de principio porque se reflexiona sobre la relación entre sistema formal del Derecho y el sistema empírico del Derecho. Entre estos dos sistemas existe una relación parecida a la dada entre un lenguaje formal y un lenguaje natural. Ambos, de hecho, son conjuntos infinitos de expresiones posibles; ambos distinguen, con base, sus propias reglas de formación, las cadenas bien formadas de las otras cadenas, ambos tienen reglas de derivadibilidad de una cadena a partir de los axiomas.

Existe sin embargo una diferencia intrínseca entre los dos. En el lenguaje formal la gramática es un a priori en relación a la expresión lingüística: las cadenas del lenguaje formal se construyen según reglas dadas; éstas pueden ser ampliadas, ampliando así la descrivibilidad del lenguaje, pero queda en el interior del sistema el retrogusto de limitación, de universo sí infinito, pero finitamente describible.

En el lenguaje natural, en cambio, la gramática es un a posteriori del hecho lingüístico: convierte en reglas formales las reglas seguidas y adquiridas en el uso, se modifica gracias al modificarse de la realidad. Las reglas vienen después de la expresión y no vinculan las expresiones posibles.

Cuando intentamos aferrar un lenguaje natural e intentamos describirlo formalmente, nos valemos de reglas context-sensitive - es decir de reglas susceptibles a los contextos-, de tal forma que las mismas fórmulas produzcan fórmulas diferentes, respetando así la diferencia entre los contextos en los cuales se ubican.

Contrariamente, cuando queremos generar un lenguaje formal a partir de sus reglas gramaticales, nos damos cuenta que las reglas de transformación son reglas esencialmente, si no exclusivamente, context-free, indican transformaciones de cadenas que se llevan a cabo independientemente del contexto en los cuales se colocan. Naturalmente las gramáticas context-free pueden ser muy articuladas, generar lenguajes infinitos y complejos de largo alcance. Sin embargo es como si el contexto no existiera en la generación de palabras y frases, como si el lenguaje generado estuviera ya intrínsecamente depurado de cualquier contextualización.

Y bien, yo pienso que un riesgo del sistema axiomático propuesto por FERRAJOLI está en su acentuación en el lenguaje formal con reglas esencialmente independientes a los contextos, que hacen que el sistema sea intrínsecamente cerrado, si bien en expansión y potencialmente ampliable. 
En la comparación entre el Derecho descrito formalmente a través de sus 16 postulados y de sus 276 definiciones y el Derecho empírico, esta limitación parece delinearse.

Es verdad que FERRAJOLI trata de evitar este riesgo acentuando la potenciabilidad inventiva y aclarando que el lenguaje formal propuesto es de tipo «observativo», en el que términos extralógicos designan objetos y procesos observables - y no de tipo teórico- en el que existen signos lógicos y términos no definibles explícitamente a través de términos observativos. Además él acentúa el hecho que en las disciplinas jurídicas los lenguajes observados son dos correspondientes a las dos interpretaciones que se pueden dar a la teoría: normativista y realista y de esta manera él se cautela, preventivamente, delante de la posibilidad que su axiomatización del lenguaje normativo sea tildada de un «puro formalismo» y, entonces, de lenguaje puramente teórico.

Pese a ello no escapa al riesgo de que el sistema designado y formalizado tenga dificultades de apertura delante de la contingencia de la mutabilidad de la historia y de la complejidad social, delante de las nuevas reglas que puedan llegar a establecerse. Las perseguirá, tratará de ponerlas en el interior, de llevarlas al propio sistema, ampliando y modificando, si se hace necesario, definiciones y axiomas y por lo tal, revisando todas las consecuencias que tales cambios determinan. Pero la relación entre sistema y realidad aparece siempre más compleja y no limitable.

El último punto que deseo subrayar proviene de las fuentes del Derecho que en sistemas europeos influenciados por el contacto con los sistemas del common law se limitan cada vez menos a las normas y se abren a la jurisprudencia. El conjunto de caseslaw constituyen una fuente normativa y sin embargo por eso mismo el control de adecuación formal es de por sí más complejo de cuanto no lo sea para las normas. Ley y sentencia, de hecho, tienen dos funciones diferentes cuando se les considera como elementos constructores del implanto lógico del Derecho: como si el primero se refiriera a la lógica y el segundo a la eurística, el primero parece pertenecer al ámbito de lo definible, el segundo al ámbito de lo que es posible.

Con una analogía arriesgada creo que las relaciones entre teoría formal del Derecho, y derecho actuado sean como aquellas que se establecen en lógica, al menos en la lógica del post Turig, entre ámbito de lo definible, de lo computable y de lo implementable.

En el ámbito de lo definible es la abstracta coherencia lógica de los objetos definidos lo que suscita interés: se puede hablar libremente de objetos infinitos y de asertos atemporales; lo que cuenta es que valga el principio de no contradicción, es decir que no puedan ser al mismo tiempo $A$ y no $A$.

El ámbito de lo computable, así como nos lo expuso A. TuRIG y como los lógicos de las funciones recursivas lo desarrollarán después, pide no sólo la coherente definibilidad de los objetos sino la existencia de un procedimiento que dé una definición constructiva.

Esta construcción es esencialmente teórica: no responde a límites de espacio o a consideraciones temporales, porque lo que es esencial es su descrivibilidad finita. 
El ámbito de lo implementable es, en cambio, el de la factibilidad de los procedimientos descritos, de su uso concreto.

Bien, yo creo que si la lógica de lo implementable es aquella que guía las elecciones, las decisiones y las concretizaciones de las elecciones normativas, es la lógica de lo computable, en el sentido ya mencionado, la que constituye el eje del Derecho como sistema regulador de una dinámica compleja y mutable, como lo es la dinámica social. Los sistemas formales, axiomáticos, a su vez dan indicaciones y guían al Derecho, pero temo que aparten a la lógica de lo definible y como tal pueden ser cercanos, pero irreduciblemente diferentes a la lógica de lo computable.

Es cierto que sin la lógica de lo definible los sistemas de lo computable no existirían o no tendrían un criterio lógico constitutivo, sin embargo no son reconducibles a ésta.

Principia Iuris es una obra in fieri, abierta, es un manifiesto programático. Pretendía tal vez ser un trabajo definitivo y definitorio, pero en cambio nos desafía no solamente cuando nos pide verificar la lógica existente sino sobre todo cuando delinea un horizonte conceptual en el cual ubicar una actividad normativa creíble.

(Traducción de Anna María Forero) 
